\title{
A DESIGN METHOD OF ADAPTIVE CONTROLLER BASED ON ROBUST DYNAMIC CERTAINTY EQUIVALENCE
}

\author{
Hiromitsu Ohmori
}

\author{
Department of Electrical Engineering, Keio University, Yokohama 223, Japan \\ Ph. +81 (45) 563 1141, ext. 3362, Fax: +81 (45) 563 2773, E-mail: ohm@sano.elec.keio.ac.jp
}

\begin{abstract}
In this paper we design a robust model reference adaptive control (RMRAC) scheme for the single input, single output, continuous time LTI plants of arbitrary relative degree in the presence of unknown deterministic disturbances and unmodeled dynamics. The design is based on robust dynamic certainty equivalence (R-DyCE) which is from the key idea of Morse's DyCE adaptive controller [3]. There are two main feature of the proposed RMRAC scheme: (1) the use of a fixed compensator in the error feedback loop to attenuate the effect of disturbances on the tracking error and to achieve robust stability in the presence of unmodeled dynamics, and (2) the use of a high-order estimator with $\sigma$-modification to ensure the global stability of the adaptive system.
\end{abstract}

\section{Introduction}

Recently two new approaches to adaptive control have been reported. The first approach uses an adaptive backstepping scheme [1], [2], and the second uses a high-order estimator [3]-[6]. In [1], [2], the authors treat the control of linear plants with unknown parameters as a nonlinear problem. In [3], a dynamic certainty equivalent (DyCE) adaptive controller is introduced. It has been shown that this type of controller can also be used to stabilize certain types of nonlinear plants having non-global Lipschitz nonlinearities [4]. In [5], [6], Ortega obtains explicit bounds on the $\mathcal{L}_{\infty}$ and $\mathcal{L}_{2}$ norms of the tracking error for DyCE adaptive controller to evaluate their transient performance. It is shown in [6], that by adding an adaptation gain in the high-order estimator, $L_{2}$ norm of the tracking error can be made arbitrarily small without increasing its $\mathcal{L}_{\infty}$ norm. This is an improvement on the results of [1], where these performance measures must be traded off in the selection of the adaptation speed.

A key assumption in the design of all the above contollers is that the plant dynamics, though unknown, is exactly representable by a member of a specified class of models. However, in practice, the plant dynamics can never be perfectly described in this way, i.e. every such model of the plant has some unmodeled dynamics. Thus stability of the control scheme and a satisfactory performance in the presence of unmodeled dynamics are two very important issues in any practical application.
In this paper we design a robust model reference adaptive control (RMRAC) scheme for the single input, single output, continuous time LTI plants of arbitrary relative degree in the presence of unknown deterministic disturbances and unmodeled dynamics. The design is based on the key idea of Morse's DyCE adaptive controller [3], [6]. There are two main feature of the proposed RMRAC scheme: (1) the use of a fixed compensator in the error feedback loop to attenuate the effect of disturbances on the tracking error and to achieve robust stability in the presence of unmodeled dynamics, and (2) the use of a high-order estimator with $\sigma$ -modification to ensure the global stability of the adaptive system. In [7], .[8], we had presented another RMRAC scheme that used the traditional certainty equivalence $(C E)$ concept together with a fixed compensator to attenuate the effect of disturbances and/or modeling errors on the tracking error. The present scheme improves on these results by using a high-order estimator to obtain a better transient performance as well. Finally we mention that in [7]-[12], adaptive control schemes based on the traditional CE (not DyCE) concept have been introduced to improve the transient performance.

\section{Problem statement}

The control objective is to track a reference signal $y_{M}(t)$ with the output $y(t)$ of an LTI plant in the presence of deterministic disturbances and unmodeled dynamics.

We consider the single input, single output, continuous LTI plants described by the input-output relationship

$$
y(t)=\left(1+W_{\Delta}(s) \Delta(s)\right)^{-1}[G(s) u(t)+d(t)]
$$

where $G(s)$ is the modeled part of the plant, i.e. the nomial plant:

$$
\begin{aligned}
& G(s):=\frac{b_{m} B[s]}{A[s]}, \\
& A[s]:=s^{n}+\sum_{i=0}^{n-1} a_{i} s^{i}, B[s]:=s^{m}+\sum_{i=0}^{m-1} b_{i} s^{i},
\end{aligned}
$$

$y(t), u(t)$ and $d(t)$ are respectively the ouput, the input and the disturbance; and $W_{\Delta}(s) \Delta(s)$ part contains the modeling error. The nomial plant transfer function coefficients $a_{t}$ 's and $b_{i}$ 's are considered unknown. We make the following standard assumptions about the plant:

Assumption 2.1 (assumptions about the plant):

(A1) Polynomials $A[s]$ and $B[s]$ are coprime.

(A2) The nominal plant order $\mathrm{n}$ and relative degree 
$\rho(=n-m) \geq 1$ are known.

(A3) The zeros of the polynomial $B[s]$ lie strictly inside the complex left half plane.

(A4) The sign of high-frequency gain is known. We will assume that $\operatorname{sgn}\left(b_{m}\right)>0$. The results in the paper can be modified in a straightforward manner to cover the case of $\operatorname{sgn}\left(b_{m}\right)<0$. Furthermore both an upper bound and a lower bound on $b_{m}$ are known such that $0<\underline{b_{m}} \leq b_{m} \leq \overline{b_{m}}$.

(A5) The disturbance $d(t)$ is uniformly bounded, i.e. $|d(t)| \leq \bar{d}, \forall t$

(A6) In the unmodeled dynamics part, $W_{\Delta}(s)$ is a known, fixed stable transfer function and $\Delta(s)$ is a variable stable transfer function such that, $W_{\Delta}(s) \Delta(s)$ is strictly proper and $\|\Delta\|_{\infty} \leq 1$, i.e. $|\Delta(j \omega)| \leq 1, \quad \forall \omega \in \Re$.

We also make the following assumptions on the reference signal $y_{M}(t)$ :

Assumption 2.2 (assumptions about the reference signal):

(A7) The reference signal $y_{M}(t)$ is the output of a reference model

$y_{M}(t)=G_{M}(s) r(t)$

where

$$
\begin{aligned}
& G_{M}(s):=\frac{B_{M}[s]}{A_{M}[s]}, \\
& A_{M}[s]:=s^{n_{M}}+\sum_{i=0}^{n_{M}-1} a_{M i} s^{i}, B_{M}[s]:=\sum_{i=0}^{m_{M}} b_{M i} s^{i} .
\end{aligned}
$$

(A8) Polynomial $A_{M}[s]$ is stable.

(A9) The signal $r(t)$ is uniformly bounded and piecewise continuous.

(A10) The relative degree $\rho_{M}\left(:=n_{M}-m_{M}\right)$ of $G_{M}(s)$ is greater than or equal to the relative degree of $G(s)$, i.e. $\rho_{M} \geq \rho$.

With tracking error $e(t)$ defined as

$$
e(t):=y(t)-y_{M}(t) \text {, }
$$

the RMRAC design problem can be sated as follows: design a control input so that tracking error $e(t)$ becomes as small as possible as $t \rightarrow \infty$.

\section{Control Structure}

In this section we propose a control structure. Our control structure includes a fixed compensator that not only reduces the effect of disturbance but also gurantees strict positive realness of the error transfer function in the presence of unmodeled dynamics.

To motivate this control structure, we first need to rewrite the plant (2.1) in a different form:

$$
C[s] y(t)=b_{m}\left[u(t)-\bar{\theta}^{T} \bar{\zeta}(t)\right]+\eta(t),
$$

where

$$
\overline{\boldsymbol{\theta}}:=\left[-b_{R(n-2)}, \ldots,-b_{R 0},-s_{n-1}, \ldots,-s_{0}\right]^{T} \in \Re^{2 n-1},
$$

$$
\begin{aligned}
& \bar{\zeta}(t):=\left[\frac{s^{n-2}}{H} u, \ldots, \frac{1}{H} u, \frac{s^{n-1}}{H} y, \ldots, \frac{1}{H} y\right]^{T}, \\
& \eta(t):=\frac{A[s] R[s]}{H[s]}\left[d(t)-W_{\Delta}(s) \Delta(s) y(t)\right], \\
& C[s]:=s^{\rho}+\sum_{i=0}^{\rho-1} c_{i} s^{i},(\text { stable polynomial), } \\
& H[s]:=s^{n-1}+\sum_{i=0}^{n-2} h_{i} s^{i}, \text { (stable polynomial), }
\end{aligned}
$$

and elements of $\bar{\theta}$ are defined from the following Diophantine equation and polynomials:

$$
\begin{aligned}
& C[s] H[s]=A[s] R[s]+b_{m} S[s], \\
& R[s]:=s^{\rho-1}+\sum_{i=0}^{\rho-2} r_{i} s^{i}, \quad S[s]:=\sum_{i=0}^{n-1} s_{i} s^{i}, \\
& B_{R}[s]:=R[s] B[s]-H[s]:=\sum_{i=0}^{n-2} b_{R i} s^{i} .
\end{aligned}
$$

With this rewriting of the plant, the tracking error can be seen to satisfy the following:

$$
\begin{aligned}
C[s] e(t)= & b_{m}\left[u(t)-\overline{\boldsymbol{\theta}}^{T} \bar{\zeta}(t)\right]+\eta(t)-C[s] y_{M}(t) \\
& =b_{m}\left[u-\overline{\boldsymbol{\theta}}^{T} \bar{\zeta}-\frac{1}{b_{m}}\left(C y_{M}-F e\right)-\frac{1}{b_{m}} F e\right]+\eta \\
& =b_{m}\left[u(t)-\theta^{T} \zeta(t)\right]-F(s) e(t)+\eta(t)
\end{aligned}
$$

then

$$
C_{F}(s) e(t)=b_{m}\left[u(t)-\theta^{T} \zeta(t)\right]+\eta(t)
$$

where

$$
\begin{aligned}
& \boldsymbol{\theta}:=\left[\begin{array}{c}
b_{m}{ }^{-1} \\
\overline{\boldsymbol{\theta}}
\end{array}\right], \quad \zeta(t):=\left[\begin{array}{c}
C[s] y_{M}(t)-F(s) e(t) \\
\bar{\zeta}(t)
\end{array}\right], \\
& C_{F}(s):=C[s]+F(s)
\end{aligned}
$$

and $F(s):=N[s] / M[s]$ is any proper transfer function which will be called the fixed compensator and its design is considered in Section 4. The parameter $\theta$ is unknown and hence we propose the following DyCE control law:

$$
u(t)=C_{F}(s)\left[\hat{\boldsymbol{\theta}}(t)^{T} \xi(t)\right], \quad \xi(t):=\frac{1}{C_{F}(s)} \zeta(t)
$$

where $\hat{\theta}(t)$ is an estimate of the parameters $\theta$. Note that the proposed control law reduces to the conventional DyCE based MRAC if $F(s)=0$.

Remark 3.1 (1) Since the relative degree of $1 / C_{F}(s)$ is $\rho$, it can be seen from (3.13) that the implementation of the control law requires first $\rho$ derivatives of $\hat{\theta}(t)$ and $\xi(t)$. Note, however that the needed derivatives of $\xi(t)$ are readily available without differentiation (see (3.13)). But the derivatives of parameter estimates $\hat{\theta}(t)$ are not available in standard estimators. This difficulty is overcome by using the high-order estimator introduced by Morse, [3], that calculates the derivatives of parameter estimates. The highorder estimator is described in Section 5.

Theorem 3.1 If the polynomials $C[s], N[s]$ and $M[s]$ are chosen such that both the polynomials $C[s] M[s]+N[s]$ and $M[s]$ are stable, then the error system can be characterized as follows:

$$
\begin{aligned}
e(t) & =\Phi(s)\left[b_{m} \tilde{\theta}(t)^{T} \xi(t)+\varepsilon(t)\right]+\Phi_{d}(s) d+\Phi_{M}(s) y_{M} \\
& =\Phi(s)\left[b_{m} \tilde{\theta}(t)^{T} \xi(t)+w(t)+\varepsilon(t)\right]
\end{aligned}
$$


where

$$
\begin{aligned}
& \tilde{\theta}(t):=\hat{\theta}(t)-\theta, \\
& \Phi(s):=\frac{1}{1+T_{F}(s) W_{\Delta}(s) \Delta(s)}, \\
& \Phi_{d}(s):=\frac{T_{F}(s)}{1+T_{F}(s) W_{\Delta}(s) \Delta(s)}, \\
& \Phi_{M}(s):=\frac{-T_{F}(s) W_{\Delta}(s) \Delta(s)}{1+T_{F}(s) W_{\Delta}(s) \Delta(s)}, \\
& T_{F}(s):=\frac{A[s] R[s]}{C_{F}(s) H[s]} \\
& w(t):=T_{F}(s) d(t)-T_{F}(s) W_{\Delta}(s) \Delta(s) y_{M}(t),
\end{aligned}
$$

and $\varepsilon(t)$ is some exponentially decaying term due to the initial conditions of the plant and the control filters.

(Proof:) As the proof can be established from straight calculation, it is omitted.

Remark 3.2 (robust stability) Since $\operatorname{sgn}\left(b_{m}\right)>0$, it is well known that the robust stability of the adaptive control system requires that the error transfer function $\Phi(s)$ be strict positive real (SPR). Notice that in the absence of modeling uncertainties, i.e. $\Delta(s)=0, \Phi(s)$ equals 1 and is thus SPR despite the presence of fixed compensator. Also note that in the absence of fixed compensator $F(s), \Phi(s)$ is not always SPR due to the unmodeled dynamics $\Delta(s)$. On the other hand, with the fixed compensator present, the error transfer function $\Phi(s)$ is also biproper and can be made SPR by proper design of the fixed compensator $F(s)$. Section 4 describes the design of the fixed compensator in detail. Intuitively, making $|M(s)|$ small at appropriate frequencies makes $\Phi(s)$ close to 1 and thus SPR.

Remark 3.3 (disturbance rejection) In the absence of the fixed compensator $F(s)$, the zeros of the disturbance transfer function $\Phi_{d}(s)$ can not be designed well, since fixing $C[s]$ and $H[s]$ automatically fixes $R[s]$. However, with the fixed compensator present, the zeros of $\Phi_{d}(s)$ can be designed via the choice of $M(s)$ to achieve the disturbance rejection.

\section{Design Scheme of Fixed Compensator}

In this section we consider the design problem of the fixed compensator. We show in particular that the design problem of the fixed compensator $F(s)$ reduces to the problem of designing a stable controller that internally stabilizes $1 / C[s]$ and optimizes a performance index.

Theorem 3.1 requires that $F(s)$ be designed such that (R1) $C[s] M[s]+N[s]$ is stable, (R2) $M[s]$ is stable. Furthermore, for robust performance we require that $F(s)$ be designed such that (R3) $\Phi(s)$ is SPR, (R4) $\Phi_{d}(s)$ attenuates the effect of the disturbance $d(t)$, and (R5) $\Phi_{M}(s)$ attenuates the effect of the reference signal $y_{M}(t)$. Clearly, requirements (R1) and (R2) ask for designing $F(s)$ such that the feedback system involving $F(s)$ and $1 / C[s]$ be internally stable (see Figure 1).

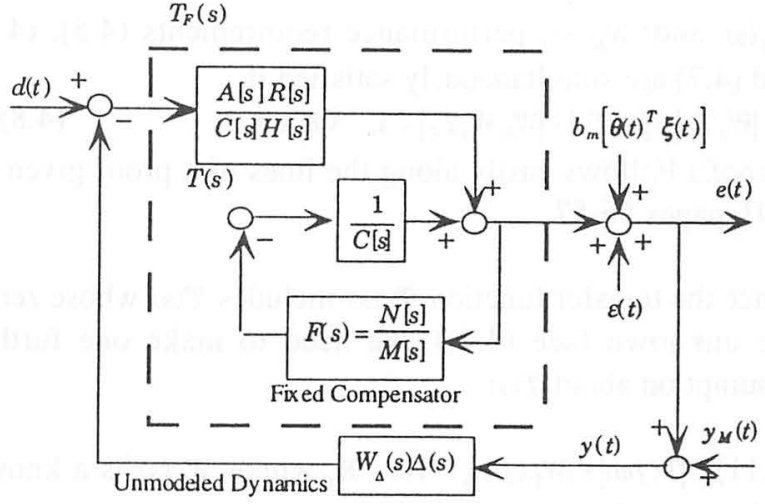

Figure 1 Design of fixed compensator

Since $1 / C[s]$ is a stable system, it follows from the well known Youla parametrization that any such $F(s)$ can be expressed as

$$
F(s)=\frac{Q(s)}{1-\frac{1}{C[s]} Q(s)}
$$

where $Q(s)$ is a stable, proper, real-rational function. Now with $U(s)$ defined as

$$
U(s):=\frac{C[s]}{C[s]-Q(s)}
$$

it follows that the transfer function $T_{F}(s)$ can be rewritten as (see (3.19), (4.1), and (4.2))

$$
T_{F}(s)=T(s) \frac{1}{U(s)},
$$

where

$$
T(s):=\frac{R[s] A[s]}{C[s] H[s]}
$$

This parameterization reduces the choice of $F(s)$ satisfying requirements (R1) and (R2) to an appropriate choice of $U(s)$. The following lemma dereives the further constraint induced by the requirement (R3).

Lemma 4.1 Let $\Phi(s)$ be as given in (3.16). Then the requirement that $\Phi(s)$ be SPR is satisfied if the requirement (R1) is satisfied and the following condition holds:

$$
\left\|W_{\Delta} T_{F}\right\|_{\infty}<1
$$

(Proof:) Follows easily along the lines of a proof given in [13].

Requirements (R4) and (R5), further demand that the following conditions hold for all possible $\Delta(s)$,

$$
\begin{aligned}
& \left\|W_{d} \Phi_{d}\right\|_{\infty}=\left\|\frac{W_{d} T_{F}}{1+T_{F} W_{\Delta} \Delta}\right\|_{\infty}<1 \\
& \left\|W_{M} \Phi_{M}\right\|_{\infty}=\left\|\frac{W_{M} W_{\Delta} T_{F}}{1+T_{F} W_{\Delta} \Delta}\right\|_{\infty}<1
\end{aligned}
$$

where $W_{d}(s)$ and $W_{M}(s)$ are weighting outer functions and are chosen based upon the a priori information on the disturbance $d(t)$ and the reference output $y_{M}(t)$ respectively. The following lemma then gives a sufficient condition that ensures simultaneous satisfaction of all the robust performance requirements (R1)-(R5).

Lemma 4.2 For given weighting outer functions $W_{\Delta}(s)$, 
$W_{d}(s)$ and $W_{M}(s)$, performance requirements (4.5), (4.6) and (4.7) are simultaneously satisfied if

$\left|W_{\Delta} T_{F}\right|+\left|W_{d} T_{F}\right|+\left|W_{M} W_{\Delta} T_{F}\right|<1, \quad \forall \omega \in \Re$.

(Proof:) Follows easily along the lines of a proof given in [14], pages 56-57.

Since the transfer function $T_{F}(s)$ includes $T(s)$ whose zeros are unknown (see (4.4)), we need to make one further assumption about $T(s)$

(A11) $|T(j \omega)| \leq\left|W_{T}(j \omega)\right|, \quad \forall \omega \in \Re$, where $W_{T}(s)$ is a known stable outer function.

With this assumption in place, the performance requirement (4.8) simplifies to the following

$$
\left|W_{U}(j \omega) \frac{1}{U(j \omega)}\right|<1, \quad \forall \omega \in \Re
$$

where

$$
\left|W_{U}\right|:=\left(\left|W_{\Delta}\right|+\left|W_{d}\right|+\left|W_{M} W_{\Delta}\right|\right) \cdot\left|W_{T}\right| \cdot
$$

Thus to ensure simultaneous satisfaction of all the robust performance requirements (R1)-(R5) it suffices to to consider the performance index:

$$
\left.J(U):=\| W_{U} \frac{1}{U}\right] \|_{\infty}
$$

$U(s)$ and thus $Q(s)$ is chosen so as to minimize the performance index of (4.11). Several approaches to perform such optimization have been researched. Ito et. al., [15], have given a simple algorithm for attaining low sensitivity by a stable controller based on a related Nevanlinna-Pick interpolation problem. By adopting either approach we can get a sub-optimal solution and the associated fixed compensator $F(s)$.

Remark $4.1 \mathrm{It}$ is clear that the transfer function $T_{F}(s)$ is designed to be stable. Hence from the assumptions (A5), (A8) and (A9), it follows that the filtered signal $w(t)$ defined by (3.20) is also uniformly bounded, i.e. there exists a constant $\bar{w}$ such that $|\omega(t)| \leq \bar{w}, \forall t$.

\section{Robust High-Order Estimator and Stability Analysis}

In this section we describe the high-order estimator and show the global stability of the proposed system. The proposed high-order estimator is essentially the high-order estimator of [6] with $\sigma$-modification[16]. The high-order estimator generates not only the estimated parameter vector, but also its first $\rho$ time derivatives. Suppose the degree of denominator (numerator) of $Q(s)$ chosen in the previous section be $\ell$. Then the estimator is given as:

$$
\begin{aligned}
\dot{\hat{\eta}}_{i}(t)= & -\gamma\left[\xi_{i}(t) e(t)+\sigma \hat{\eta}_{i}(t)\right], \\
& \hat{\eta}_{i}(0)=C_{F}(0) \hat{\theta}_{i}(0), \\
\dot{\hat{x}}_{i}(t)= & \lambda_{i}^{2}(t)\left[A \hat{x}_{i}(t)+b \hat{\eta}_{i}(t)\right], \\
& \hat{x}_{i}(0)=-C_{F}(0) A^{-1} b \hat{\theta}_{i}(0), \quad \hat{x}_{i}(t) \in \Re^{\bar{n}} \\
\hat{\theta}_{i}(t)=c^{T} \hat{x}_{i}(t), & \\
\lambda_{i}^{2}(t)= & 1+\mu_{0}+\mu_{1} \xi_{i}^{2}(t)
\end{aligned}
$$

where $\bar{n}:=2 p+1, \quad \xi_{i}(t)$ and $\hat{\theta}_{i}(t)$ are respectively the $i$-th elements of $\xi(t)$ and $\hat{\theta}(t), i=1,2, \ldots, 2 n, \gamma, \sigma, \mu_{0}$ and $\mu_{1}$ are positive constants to be defined later and $\left(A, b, c^{T}\right)$ is a minimal canonical realization of $1 / C_{F}(s)$ for which we have

$$
A^{-1} b=\frac{-c}{C_{F}(0)}:=-\bar{c} .
$$

Since $1 / C_{F}(s)=c^{T}(s I-A)^{-1} b$ and both the numerator and denominator of $1 / C_{F}(s)$ are stable, we also have

$$
\frac{1}{C_{F}(0)}=-c^{T} A^{-1} b>0, \quad c^{T} c=1 .
$$

Remark 5.1 From the fact that the relative degree of $1 / C_{F}(s)$ is $\rho$, we have that $c^{T} A^{k} b=0$ for $k=0,1, \ldots, \rho-2$. Consequently, it follows from (5.1) that the first $\rho$ derivatives of $\hat{\theta}(t)$ can be obtained from $\hat{x}_{i}(t)$ 's, $\hat{\eta}_{i}(t)$ 's, $\xi(t)$ and its first $\rho-1$ derivatives. Recall from Remark 3.1 that the needed derivatives of $\xi(t)$ are available without differentiation. Hence the adaptive control law of (5.1) can be implemented without differentiation.

Let $\left(A_{e}, b_{e}, c_{e}{ }^{T}, d_{e}\right)$ be a minimal realization of $\Phi(s)$, then (3.14) can be rewritten as

$$
\left\{\begin{array}{l}
\dot{v}(t)=A_{e} v(t)+b_{e} v(t) \\
e(t)=c_{e}{ }^{T} v(t)+d_{e} v(t)
\end{array}\right.
$$

where the input signal is defined by $v(t):=b_{m} \tilde{\theta}(t)^{T} \xi(t)+w(t)+\varepsilon(t)$. Note that $d_{e}=1$ since $\Phi(\infty)=1$ (see (3.16) and (A6)). Further, if $U(s)$ is chosen to satisfy the performance requirement (4.9), then $\Phi(s)$ is SPR and from Lefschetz-Kalman-Yacobovich (LKY) lemma [17] we have the existence of real, symmetric positive definite matrices $\boldsymbol{X}$ and $\boldsymbol{L}$, a real vector $\boldsymbol{q}$ and a positive constant $\delta$ such that

$$
\begin{aligned}
& A_{e}{ }^{T} X+X A_{e}=-q q^{T}-\delta L \\
& X b_{e}=c_{e}+\sqrt{2 d_{e}} q
\end{aligned}
$$

Now to obtain the error system of adaptive law (5.1), we first define the following error signals:

$$
\begin{array}{ll}
\tilde{\eta}_{i}(t):=\hat{\eta}_{i}(t)-\eta_{i}, & \eta_{i}:=C_{F}(0) \theta_{i} \\
\tilde{x}_{i}(t):=\hat{x}_{i}(t)-x_{i}, & x_{i}:=-C_{F}(0) A^{-1} b \theta_{i}
\end{array}
$$

where $\theta_{i}$ denotes the $i$ th elements of $\theta$ and $i=1, \ldots, 2 n$. Then (5.1)-(5.3) together with (5.10), (5.11) gives

$$
\begin{gathered}
\dot{\tilde{\eta}}_{i}(t)=-\gamma\left[\xi_{i}(t) e(t)+\sigma\left(\tilde{\eta}_{i}(t)+\eta_{i}\right)\right], \\
\tilde{\eta}_{i}(0)=C_{F}(0) \tilde{\theta}_{i}(0), \\
\dot{\tilde{x}}_{i}(t)=\lambda_{i}{ }^{2}(t)\left[A \tilde{x}_{i}(t)+b \tilde{\eta}_{i}(t)\right], \\
\tilde{x}_{i}(0)=-C_{F}(0) A^{-1} b \tilde{\theta}_{i}(0), \\
\tilde{\theta}_{i}(t)=c^{T} \tilde{x}_{i}(t)
\end{gathered}
$$

where in deriving the last equation we have used (5.6). The equations (3.14), (5.4) and (5.12)-(5.14) constitute the error system of the proposed system. This error system brings us to the following theorem about the global stability of the proposed system.

Theorem 5.1 Consider the system satisfies the assumptions (A1)-(A11) in closed loop with the dynamic certainty equivalent adaptive controller (3.13) with fixed compensator 
that is designed to satisfy (4.9). The controller parameters are updated with the robust high-order estimator (5.1)-(5.4) with $\mu_{0}$ and $\mu_{1}$ chosen such that

$$
\begin{aligned}
& \mu_{1}>\frac{4 \gamma^{2} n}{\beta \delta} \frac{\left\|c_{e}\right\|^{2}}{\lambda_{\min }(L)}\left\|P_{0} A^{-1} b\right\|^{2}, \\
& \mu_{0} \geq \frac{C_{F}(0)}{b_{m}}\left\|P_{1} \bar{c}\right\|^{2} \gamma \sigma
\end{aligned}
$$

where $P_{1}$ is the unique symmetric positive definite solution of $\boldsymbol{A}^{T} \boldsymbol{P}_{1}+\boldsymbol{P}_{1} \boldsymbol{A}=-\boldsymbol{I}$, and $\boldsymbol{P}_{0}$ is defined by $P_{0}:=P_{1}+b_{m} C_{F}(0) c c^{T}$. Under these conditions, for all initial conditions, the tracking error $e(t)$ converges in certain closed region and all the signals and parameters in the proposed system are bounded.

(Proof:) Consider the following quadratic function along the lines of [7]

$$
V:=\frac{1}{2} \sum_{i=1}^{2 n}\left[z_{i}{ }^{T} P_{1} z_{i}+\frac{b_{m}}{C_{F}(0)} \tilde{\eta}_{i}{ }^{2}\right]+\frac{\beta}{2} v^{T} X v:=V_{1}+V_{2}
$$

where $\beta>0$ is definded later and

$$
\boldsymbol{z}_{i}(t):=\tilde{x}_{i}(t)-\bar{c} \tilde{\eta}_{i}(t)
$$

for $i=1,2, \ldots, 2 n$. We first show that the derivative of $V_{1}(t)$ along the trajectories of the given system satisfies

$$
\begin{aligned}
\dot{V}_{1}(t) & =\sum_{i=1}^{2 n}\left[z_{i}{ }^{T}(t) P_{1} \dot{z}_{i}(t)+\frac{b_{m}}{C_{F}(0)} \tilde{\eta}_{i}(t) \dot{\tilde{\eta}}_{i}(t)\right] \\
\leq & -\frac{1}{2} \sum_{i=1}^{2 n}\left\|z_{i}(t)\right\|^{2}-\gamma(t) e(t)+\gamma(\omega(t)+\varepsilon(t)) e(t) \\
& +\frac{\gamma^{2} n}{\mu_{1}}\left\|P_{0} \bar{c}\right\|^{2} e^{2}(t)-k_{\sigma} \sum_{i=1}^{2 n} \tilde{\eta}_{i}{ }^{2}(t)+k_{\sigma} \sum_{i=1}^{2 n} \eta_{i}{ }^{2}
\end{aligned}
$$

where $k_{\eta}:=k_{\sigma}-\frac{\gamma^{2} \sigma^{2}}{2 \mu_{0}}\left\|P_{1} \bar{c}\right\|^{2}$ and $k_{\sigma}:=\frac{\gamma \sigma b_{m}}{2 C_{F}(0)}$. It is clear that $k_{\eta} \geq 0$ from (5.16). Secondly, the derivative of $V_{2}(t)$ along the trajectories of the given system satisfies

$$
\begin{aligned}
\dot{V}_{2}(t) & =\beta v^{T}(t) \boldsymbol{X}\left(A_{e} v(t)+b_{e} v(t)\right) \\
& \leq-\frac{\beta \delta}{4} v^{T}(t) L v(t)-\frac{\beta \delta}{4} \frac{\lambda_{\min }(L)}{\left\|c_{e}\right\|^{2}} e^{2}(t)+\beta k_{1} v(t) e(t)
\end{aligned}
$$

where we have defined $k_{1}:=1+\frac{\delta}{2} \frac{\lambda_{\min }(L)}{\left\|c_{e}\right\|^{2}}$. Combining (5.19) and (5.20), we have,

$$
\begin{aligned}
\dot{V}(t)= & \dot{V}_{1}(t)+\dot{V}_{2}(t) \\
\leq & -\frac{1}{2} \sum_{i=1}^{2 n}\left\|z_{i}(t)\right\|^{2}-k_{\sigma} \sum_{i=1}^{2 n} \tilde{\eta}_{i}^{2}(t)-\frac{\beta \delta}{4} v^{T}(t) L v(t) \\
& +\left(\beta k_{1}-\gamma\right) v(t) e(t)-\frac{\gamma k_{e}}{2} e^{2}(t)+\gamma \varepsilon(t) e(t) \\
& -\frac{\gamma k_{e}}{2} e^{2}(t)+\gamma \omega(t) e(t)+k_{\sigma} \sum_{i=1}^{2 n} \eta_{i}{ }^{2}
\end{aligned}
$$

where $k_{e}$ is defined as $k_{e}:=\frac{\beta \delta}{4 \gamma} \frac{\lambda_{\min }(L)}{\left\|c_{e}\right\|^{2}}-\frac{m}{\mu_{1}}\left\|P_{0} \bar{c}\right\|^{2}$. It is clear $k_{e}>0$ from (5.15). Since no constraint has been placed on the choice of $\beta$ in (5.17), except that it should be positive, we can set $\beta$ as $\beta=\gamma / k_{1}$. Then notice that $\beta k_{1}-\gamma=0$. Now (5.21) becomes

$$
\dot{V}(t) \leq-\alpha V(t)-\frac{\gamma_{e}}{2} e^{2}(t)+\gamma \varepsilon(t) e(t)+K_{0}
$$

where we have defined

$$
\begin{aligned}
& K_{0}:=k_{\sigma} \sum_{i=1}^{2 n} \eta_{i}{ }^{2}+\frac{\gamma}{2 k_{e}} \bar{w}^{2}, \\
& \alpha:=\min \left\{\frac{1}{\lambda_{\max }\left(\boldsymbol{P}_{1}\right)}, \quad \sigma \gamma, \frac{\delta \lambda_{\min }(\boldsymbol{L})}{2 \lambda_{\max }(\boldsymbol{X})}\right\} .
\end{aligned}
$$

Therefore due to the property of $\lim _{t \rightarrow \infty} \varepsilon(t)=0$ and $K_{0}<\infty$ (since $|\omega(t)| \leq \bar{w}<\infty$ (see Remark 4.1)), for some $V_{0}>0$ and all $V(t)>V_{0}$ we have $\dot{V}(t)<0$ which implies that $V \in L_{\infty}$, i.e. $z_{i}(t), \tilde{\eta}_{i}(t), v(t) \in L_{\infty}$. The global boundedness of all signals in the proposed system now proceeds along classical lines [13].

Remark 5.2 Notice from (5.22), that smaller values of $K_{0}$ lead to good asymptotic performance, while larger values of $\alpha$ lead to good transient performance. Smaller values of $K_{0}$ are achieved by small $\sigma, \gamma, \bar{w}$ and large $\mu_{0}, \mu_{1}$. Value of $\bar{w}$ depends on the optimal performance index (4.12). On the other hand, larger values of $\alpha$, to a limit, are achieved by large $\sigma$ and $\gamma$ (i.e. fast adaptation). So for satisfactory performance, we should first choose a large enough $\sigma$ and $\gamma$ to achieve good transient performance and then choose large $\mu_{0}$ and $\mu_{1}$ to achieve good asymptotic performance.

Remark 5.3 Note that the knowledge of both an upper and a lower bound on the magnitude of the high-frequency gain is needed to choose $\mu_{1}$ and $\mu_{0}$, respectivly.

\section{Simulations Results}

Consider the continous time system in the presence of unmodeled dynamics and ditrubance described by

$$
y(t)=\left(1+\frac{0.08}{0.11 s^{2}+0.0067 s+1}\right)^{-1}\left[\frac{4}{s^{2}+4 s+4} \dot{u}(t)+d(t)\right],
$$

where

$$
d(t)=\sin 3.1 t,
$$

and the referenxce model and signal are given by

$$
\begin{aligned}
& G_{M}(s)=\frac{2}{s^{2}+3 s+2}, \\
& r(t)=2 \sin 3.1 t+0.5 \sin 0.6 t+0.5 \sin 1.5 t .
\end{aligned}
$$

Then simulating the adaptive control system with

$$
C(s)=s^{2}+3 s+2, H(s)=s+3
$$

Figure 2 shows the simulation results without the fixed compensator. In this case there is no guarantee that the error system is SPR.. Figure 3 shows the results with the fixed compensator,

$$
F(s)=\frac{40 s^{2}+20 s+2.4}{s^{2}+0.00124 s+9.61} .
$$

We can berify the condition $\left\|W_{\Delta} T_{F}\right\|_{\infty} \leq 1$ in lemma 4.1. Comparing figure 2 and 3 , we can see our proposed system attain good control performance.

\section{Conclusions}

We have presented a new RMRAC scheme for plants with arbitrary relative degree in the presence of unknown deterministic disturbances and unmodeled dynamics. The 
fixed compensator in this scheme is designed from a related $H_{\infty}$ minimization problem. A high-order estimator with $\sigma$ -modification ensures the global stability of the proposed system.

\section{References}

[1] M. Krstic, I. Kanellakopoulos and P.V. Kokotovic, "A new generation of adaptive controllers for linear systems," Proc. 31 st Conf. Decision Contr., Tucson, Arizona, pp.3644-3650, 1992.

[2] M. Krstic, P.V. Kokotovic, and I. Kanellakopoulos, "Transient-performance improvement with a new class of adapitve controllers," Systems \& Control Letters, vol.21, pp.451-461, 1993.

[3] D.R. Mudgett and A.S. Morse, "High-order parameter adjustment laws for adaptive stabilization," Proc. 1987 Conf. Info. Sci. Sys., 1987, pp.314-317.

[4] A. S. Morse, "A comparative study of normalized and unnormalized tuning errors in parameter adaptive control," Int. J. Adaptive Control and Signal Processing, vol.6, pp.309-318, 1992.

[5] R. Ortega, "On Morse's On Morse's new adaptive controller: parameter convergence and transient performance," IEEE Trans. Automat. Contr., vol.38, pp.1191-1202, 1993.

[6] R. Ortega, "Transient bounds of dynamic certainty equivalent adaptive controllers," Int. J. Adaptive Control and Signal Processing, vol.7, pp.291-295, 1993.

[7] H. Ohmori and A. Sano, "Model reference adaptive control system including compensator for unknown deterministic disturbances," Trans. SICE, vol.20-10, pp.919-915, 1984. (in Japanese)

[8] H. Ohmori and A. Sano, "Design of robust adaptive control system with a fixed compensator," Robust Control, Lecture Notes in Control and Information Science, S. Hosoe (Ed.), 1992, pp 146-153.

[9] A. Datla and P. A. Ioannou, "Performance improvement versus robust stability in model reference adaptive control," Proc. 30th Conf. Decision Contr., Brighton, England, 1991, pp.748753.

[10] I.N.M. Pappadakis and S.C.A. Thomopoulos, "Modified output error method with parameter mismatch compensation for improved performance model reference adaptive control," Proc. 3Ist Conf. Decision Contr., pp.3249-3254, Tucson, Arizona, 1992, pp 3249-3254.

[11] A. Datta, "Transient Performance Improvement in Continuous-time Model Reference Adaptive Control an L Formulation," Proc. of ACC, pp.294-295, 1993.

[12] A. Datta and M. Ho, "On modifying model reference adaptive control schemes for performance improvement," Proc. 32nd Conf. Decision Contr., San Antonio, TX, 1993, pp 1093-1094.

[13] R.L. Kosut and C.R. Johnson, Jr., "An input-output view of robustness in adaptive control," Automatica, vol. 20, pp 569-581, 1984.

[14] J. C. Doyle, B. A. Francis, and A. R. tannenbaum, "Feedback Control Theory", Macmillan Publishing Company, 1992.

[15] H. Ito, H. Ohmori and A. Sano, "Design of stable controllers attaining low $H_{\infty}$ weighted sensitivity," IEEE Trans. Automat. Contr., vol. 38, pp 485-488, 1993.

[16] R. Ortega and T. Yu, "Robustness of adaptive controllers: A suvey," Automatica, vol.25, no.5, 1989.

[17] J. H. Taylor, "Strictly positive-real functions and the
Lefscchetz-Kalman-Yakubovich(LKY) lemma," IEEE Trans. Circuits and Systems, no.3, pp.310-311, 1974.

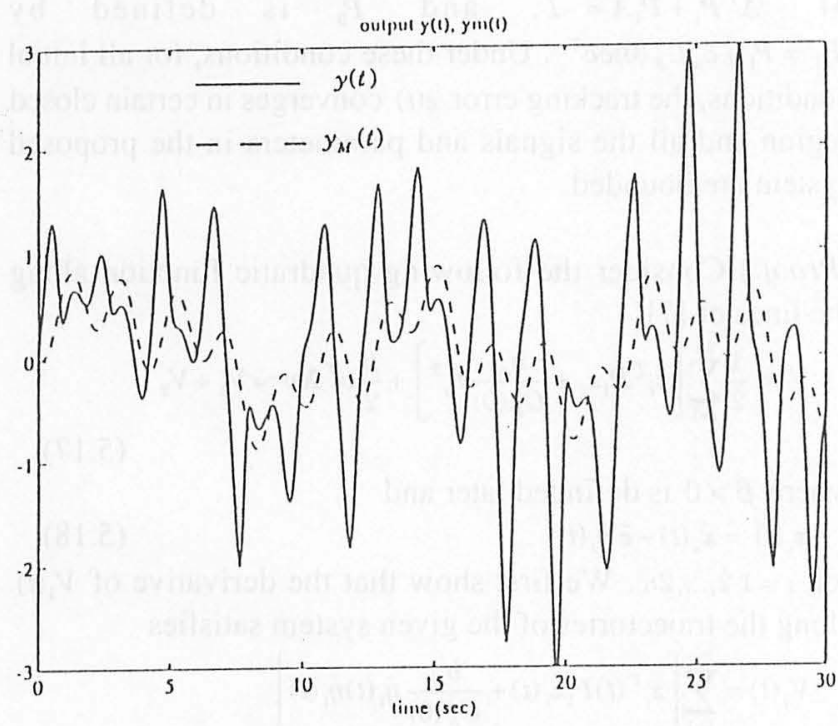

Figure 2 Simulation results in the absene of the fixed compensator.

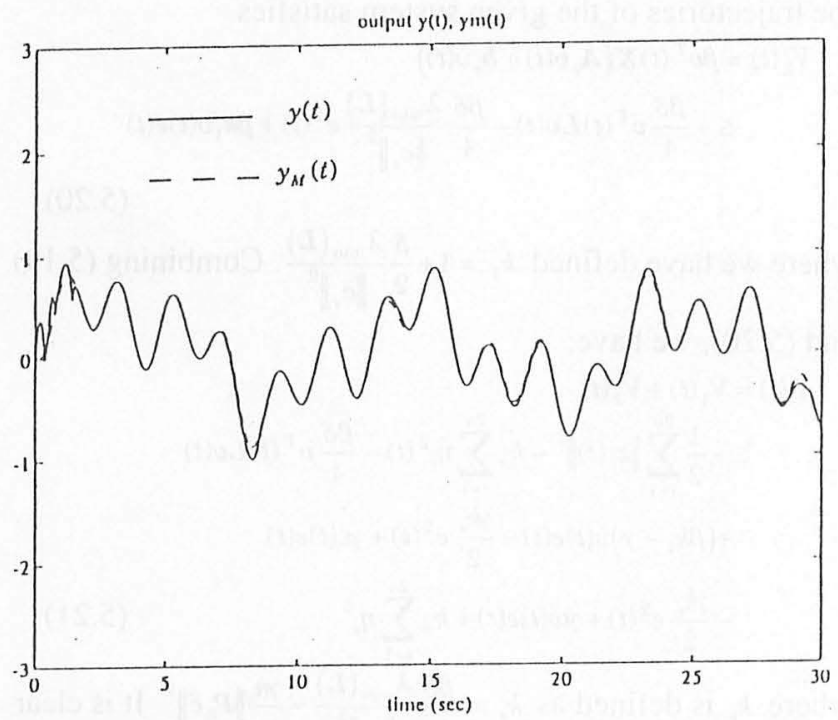

Figure 3 Simulation results in the presence of the fixed compensator. 